\title{
Microfilarial distribution of Loa loa in the human host: population dynamics and epidemiological implications
}

\author{
S. D. S. PION ${ }^{1,2 *}$, J. A. N. FILIPE ${ }^{2}$, J. KAMGNO ${ }^{1}$, J. GARDON ${ }^{1,3}$, M.-G. BASÁÑEZ ${ }^{2}$ \\ and M. BOUSSINESQ ${ }^{1,4}$ \\ ${ }^{1}$ Laboratoire mixte IRD (Institut de Recherche pour le Développement) - CPC (Centre Pasteur du Cameroun) d'Epidémiologie \\ et de Santé publique, Centre Pasteur du Cameroun, BP 1274, Yaoundé, Cameroun \\ ${ }^{2}$ Department of Infectious Disease Epidemiology, St Mary's Campus, Norfolk Place, London W2 1PG, UK \\ ${ }^{3}$ Institut de Recherche pour le Développement, UR 24 Epidémiologie et Prévention, CP 9214 Obrajes, La Paz, Bolivia \\ ${ }^{4}$ Institut de Recherche pour le Développement, Département Sociétés et Santé, 213 rue La Fayette, 75480 Paris \\ Cedex 10, France
}

(Received 16 November 2005; revised 10 fanuary 2006; accepted 10 fanuary 2006)

\begin{abstract}
SUMMARY
Severe adverse events (SAEs) following ivermectin treatment may occur in people harbouring high Loa loa microfilarial (mf) densities. In the context of mass ivermectin distribution for onchocerciasis control in Africa, it is crucial to define precisely the geographical distribution of L. loa in relation to that of Onchocerca volvulus and predict the prevalence of heavy infections. To this end, we analysed the distribution of $\mathrm{mf}$ loads in 4183 individuals living in 36 villages of central Cameroon. Mf loads were assessed quantitatively by calibrated blood smears, collected prior to ivermectin distribution. We explored the pattern of $L$. loa $\mathrm{mf}$ aggregation by fitting the (zero-truncated) negative binomial distribution and estimating its overdispersion parameter $k$ by maximum likelihood. The value of $k$ varied around $0 \cdot 3$ independently of mf intensity, host age, village and endemicity level. Based on these results, we developed a semi-empirical model to predict the prevalence of heavy $L$. loa $\mathrm{mf}$ loads in a community given its overall $\mathrm{mf}$ prevalence. If validated at the continental scale and linked to predictive spatial models of loiasis distribution, this approach would be particularly useful for optimizing the identification of areas at risk of SAEs and providing estimates of populations at risk in localities where L. loa and O. volvulus are co-endemic.
\end{abstract}

Key words: Loa loa, microfilarial aggregation, ivermectin, Cameroon.

\section{INTRODUCTION}

The African filarial worm Loa loa is well known for spectacularly migrating under the eye conjunctiva, and its association with transient oedemas called 'Calabar swelling'. Even if these signs make it one of the primary causes of consultation in the endemic areas (Boulesteix and Carme, 1986), loiasis is not regarded as a very serious disease. However, individuals harbouring high microfilaraemias may, exceptionally, develop serious spontaneous neurological or renal complications (Cauchie et al. 1965; Zuidema, 1971). More significantly, it is well known that high microfilarial ( $\mathrm{mf}$ ) loads are associated with a risk of developing neurological serious adverse events (SAEs) after treatment with the filaricidal drugs diethylcarbamazine (DEC) and ivermectin (Fain, 1978; Carme et al. 1991; Gardon et al. 1997 a ; Boussinesq et al. 1998). Thus, following ivermectin treatment, it has been demonstrated that individuals

* Corresponding author: Department of Infectious Disease Epidemiology, St Mary's Campus, Norfolk Place, London W2 1PG, UK. Tel: + 44 (0)20 7594 3622. Fax: +44(0)20 7594 3693. E-mail: s.pion@no-log.org presenting with high $\mathrm{mf}$ loads $(>8000 \mathrm{mf} / \mathrm{ml})$, and those with very high $\mathrm{mf}$ loads (>30 $000 \mathrm{mf} / \mathrm{ml}$ ) had, respectively, an increased risk of developing severe adverse reactions without neurological involvement, and SAEs (Gardon et al. 1997 a). In the context of the Community Directed Treatment with Ivermectin (CDTI) carried out in Africa, these SAEs are of crucial concern as they can lead to fatal outcomes and jeopardize the success of the African Programme for Onchocerciasis Control (APOC) (Twum-Danso, 2003).

In loiasis, many individuals do not present with microfilariae (mfs) in their peripheral blood, a phenomenon usually described as 'occult loiasis', yet they may prove to be infected because of previous history of subconjunctival worm passage. While genetic epidemiology (Garcia et al. 1999) and immunological studies (Winkler et al. 1999; Akué et al. 2002; Walker-Deemin et al. 2004) have brought useful insights into understanding the processes leading to some individuals developing $L$. loa microfilaraemia, the population dynamics of $L$. loa remains poorly documented. In a previous paper, we presented a detailed analysis of the structure of the microfilarial reservoir of $L$. loa in an endemic 
population through the study of host age- and sexspecific parasitological profiles in terms of prevalence and intensity of microfilaraemia (Pion et al. 2004). Our results indicate that the prevalence of microfilaraemia increases with age, is higher for males than females and, more unexpectedly, that, for a given level of endemicity, the mean intensity among microfilaraemic individuals remains nearly unchanged with host age.

The prevalence and intensity of an infection are but two characteristics of the distribution of parasites among hosts. In addition, parasite distributions are typically overdispersed. The degree of parasite overdispersion is a key parameter of the stability and dynamics of a host-parasite system (May and Anderson, 1978; Dobson and Hudson, 1992) and, according to some authors, it is constant and characteristic for a given host-parasite system (Bliss and Fisher, 1953; Pichon et al. 1975, 1980; Quinnell et al. 1995). However, it would be expected that the distribution of parasites per host is a dynamic property within a given host-parasite system depending, for instance, on the intensity of transmission, the age-structure of the host population, and the operation of agedependent and/or density-dependent processes (Adler and Kretzschmar, 1992; Pugliese et al. 1998). Since, to our knowledge, $\mathrm{mf}$ aggregation has never been characterized for $L$. loa, we focus, in the present paper, on the distribution of the L. loa mf loads in the human population.

Besides the population dynamics aspects, characterizing the distribution of $L$. loa $\mathrm{mfs}$ among humans may be particularly useful for assessing the proportion of the host population at risk of posttreatment SAEs. In particular, the negative binomial distribution (NBD) provides a simple relationship between the prevalence and the mean intensity of infection which depends on the magnitude and functional form of the overdispersion parameter (Anderson, 1982). This relationship has been used to investigate the distribution of helminths parasites in humans (Anderson and May, 1985; Guyatt et al. 1990; Basáñez and Boussinesq, 1999), wildlife hosts (Shaw and Dobson, 1995; Shaw et al. 1998), and vectors (Cheke et al. 1982; Renz, 1987; Basáñez et al. 1995). If the overdispersion parameter can be determined for $L$. loa, it would be possible, in principle, to estimate the mean $\mathrm{mf}$ load in a community given its prevalence. This approach has been used successfully for Onchocerca volvulus in humans (Basáñez et al. 2002) and vectors (Basáñez et al. 1998).

Since a predictive spatial model for prevalence of L. loa microfilaraemia from environmental data obtained by remote sensing has been developed and validated (Thomson et al. 2004), prevalence estimates can easily be obtained for the whole distribution area of the parasite. If the NBD model were also validated, merging the results obtained by the Thomson et al. model with a well-defined relationship between community prevalence of microfilaraemia and prevalence of heavy infections, would provide a useful tool to aid SAEs surveillance in CDTI campaigns.

In the present study, we explore the patterns of L. loa $\mathrm{mf}$ aggregation in endemic populations, and develop and test a model to predict the prevalence of heavy $L$. loa $\mathrm{mf}$ loads in a community given its $\mathrm{mf}$ prevalence.

\section{PATIENTS AND METHODS}

\section{Study area and parasitological surveys}

The study areas and the methods used for selecting and examining subjects have been previously described (Gardon et al. 1997 a ; Boussinesq et al. 2001; Pion et al. 2004). Briefly, the data analysed in the present paper were collected as part of a trial conducted in 1995-1996 in the Lékié Division (Central Province, Cameroon) to evaluate the incidence of L. loa related post-ivermectin SAEs and to identify risk factors associated with the latter. During this trial, 4183 subjects aged $\geqslant 15$ years were examined in 36 communities. This age group was chosen because, at the time of this trial, all the SAEs reported so far had occurred in individuals $\geqslant 15$ years.

From each consenting individual, a blood sample was collected by finger-prick, between 10.00 and $16.00 \mathrm{~h}$, in a non-heparinized capillary tube, and calibrated thick blood films were immediately prepared, using $50 \mu \mathrm{l}$ of blood. Each Giemsa-stained smear was then examined under a low-power microscope and all the $L$. loa $\mathrm{mfs}$ present on the slide were identified and counted. All the persons examined had been questioned as to whether they had received any antifilarial treatment previously, and the data from those few who had been treated during the last 5 years were discarded from analysis.

\section{Statistical analysis}

Method to assess overdispersion. Various methods to assess the degree of parasite contagion or aggregation have been advocated in the literature, among which the variance to mean ratio (VMR) investigates discrepancy from the Poisson or random distribution $(\mathrm{VMR}=1)$, and the index of discrepancy measures departures from the uniform distribution (all hosts harbour the same number of parasites) (Poulin, 1993; Poulin and Morand, 2000). We chose to assess aggregation through the parameter $k$ of the NBD fitted to observed $\mathrm{mf}$ distributions in population strata as defined in the following section. However, during preliminary analyses, the NBD model, when fitted to the complete observed distributions of $\mathrm{mf}$ densities (including zero densities), did not provide satisfactory fits, whereas the zero-truncated NBD model provided adequate fits. Thus, assuming that the zero count class may not be reliable because only 
$\sim 60 \%$ of the infected population would be genetically predisposed to present with microfilaraemia (Garcia et al. 1999), and that some individuals may be false-negatives (due to the lack of sensitivity of the blood film method when microfilaraemia is low), we chose the zero-truncated NBD (tNBD) model (Pichon et al. 1980; Grenfell et al. 1990).

Estimates of $k$ (and corresponding variance) were obtained using the maximum likelihood method (MLM) proposed by Sampford (1955) and confidence intervals were obtained by bootstrapping (1000 simulations for each stratum). The fits to tNBD and calculations of confidence intervals were performed using Stata 9.0. Goodness of fit was tested using $\chi^{2}$ tests with the number of degrees of freedom equal to the number of frequency classes -3 (Elliott, 1977).

Patterns of microfilarial aggregation with host age and sex by level of endemicity. Investigation of parasite overdispersion with age and transmission intensity has been used to obtain insights into the possible operation of age- or parasite density-related processes regulating population dynamics (Anderson and Gordon, 1982; Pacala and Dobson, 1988; Fulford et al. 1992; Woolhouse et al. 1994; Das et al. 1995; Filipe et al. 2005). We classified the villages according to 3 endemicity levels based on the prevalence of microfilaraemia in the population aged $\geqslant 15$ years, as a proxy for transmission intensity. These levels were: low endemicity $(<25 \% \mathrm{mf}$ prevalence), moderate endemicity (25-34.9\%) and high endemicity $(\geqslant 35 \%)$. In each of these categories, the populations were subsequently sorted by sex and age according to the following age classes: 15-19, $20-29,30-39,40-49,50-59$ and $\geqslant 60$ years. The total population was thus divided in 36 different strata (3 prevalence classes $\times 2$ sexes $\times 6$ age classes). We estimated $k$ for each separate stratum. We then tested whether $k$ varied by group using a linear regression of $k$ on age-sex-endemicity group; such a method has been used to investigate aggregation patterns of Schistosoma haematobium in human populations (Woolhouse et al. 1994).

Predicting prevalence of heavy infection given $m f$ prevalence. We aimed at developing a model to predict the prevalence of heavy L. loa mf loads in a community given the prevalence of microfilaraemia in those aged $\geqslant 15$ years in such a community. To this end, we considered the village as the epidemiological unit, so this part of the analysis was conducted at community level.

Let $X_{i}$ denote the random variable equal to the $\mathrm{mf}$ count in an individual aged $\geqslant 15$ years old and living in community $i ; x_{i}$ the actual value of $X_{i} ; \pi_{i}$ the overall prevalence of microfilaraemia in those aged $\geqslant 15$ years in community $i$ (i.e. $\pi_{i}=\operatorname{Prob}\left(X_{j}>0\right)$, and $T$ the microfilaraemia threshold above which an individual is considered to have heavy infection.
Then, following the results obtained in the first part of the analysis, we assume that, in a given village $(i)$, the frequency distribution of $\mathrm{mf}$ counts in those microfilaraemic follows a truncated NBD with parameter $M_{i}$, the mean $\mathrm{mf}$ intensity, and $k_{i}$, the overdispersion index, i.e.

$X_{i} \sim \operatorname{tNBD}\left(M_{i}, k_{i}\right)$. Thus, the proportion of people in community $i$ presenting with more than $T \mathrm{mf} / \mathrm{ml}$ is :

$P_{i}\left(X_{i}>T\right)=\left[1-\sum_{x_{i}=1}^{T} p_{i}\left(x_{i}\right)\right] \pi_{i}$

Equation (1)

Explicitly, the tNBD of $\mathrm{mf}$ counts is given by:

$p_{i}(x)=\frac{\Gamma\left(x+k_{i}\right)}{\Gamma\left(k_{i}\right) x !} \frac{q_{i}^{x}\left(1-q_{i}\right)^{k_{i}}}{\left[1-\left(1-q_{i}\right)^{k_{i}}\right]}$

where $q_{i}=\frac{M_{i}}{M_{i}+k_{i}}$ and $\Gamma$ is the gamma function.

Our aim was to render expression (1) exclusively in terms of $\pi_{i}$. For this purpose, we modelled, on the one hand, $M_{j}$ as a function of $\pi_{i}$, and, on the other hand, $k_{i}$ as a function of $\pi_{i}$.

(i) Relationship between $M_{i}$ and $\pi_{i}$

As the simplest possible approximation and motivated by inspection of the data, we assumed a linear relationship, across communities, between the mean microfilarial load and the microfilarial prevalence in those aged $\geqslant 15$ years,

$M_{i}=A_{i} \pi_{i}$

Equation (2)

(ii) Relationship between $k_{i}$ and $M_{i}$

Parameter $k_{i}$ was estimated for each separate village $i$ using the maximum likelihood method described in the first part of the analysis. Then, parameter $k_{i}$ was included in equation (1) using 2 alternative functional forms: a constant value $k_{c}=0 \cdot 3$ (the mean $k$ value obtained either in the 'per age and sex stratum' analysis or in the 'per village' analysis of the current data, see Results section), and a log-linear relationship with mean $\mathrm{mf}$ intensity, $k_{i}=\alpha+\beta \log \left(M_{i}\right)$. This model has been chosen because $M_{i}>0$. In this latter model, we also used the relationship between $M_{i}$ and $\pi_{i}$ derived from equation (2). The empirical relationships between $M_{i}$ and $\pi_{i}$, and between $k_{i}$ and $M_{i}$ were fitted to the current study data using the least squares method, analogous to the linear regression approach used above.

We applied expression (1) to 2 different threshold values: $T=8000 \mathrm{mf} / \mathrm{ml}$ and $30000 \mathrm{mf} / \mathrm{ml}$. The first value corresponds to the threshold above which there is a significant increase in the relative risk of occurrence of functional impairment following ivermectin treatment, and the second threshold, to the value above which the risk of occurrence of serious neurological reactions is increased (Gardon et al. 1997 a). Deviation of these predictions from the observed data was assessed using $\chi^{2}$ tests. 
Table 1. Number of individuals presenting with Loa loa microfilaraemia among the total number of subjects examined and arithmetic mean of the positive microfilarial loads (in parentheses) for each sex-, age- and endemicity (measured as the $\mathrm{mf}$ prevalence) category in the 36 villages of the Lékié division surveyed in 1995-1996

(A total of 8,15 and 13 villages were respectively grouped in the $<25 \%, 25-35 \%$ and $\leqslant 35 \%$ endemicity categories.)

\begin{tabular}{|c|c|c|c|c|c|c|}
\hline \multirow{2}{*}{$\begin{array}{l}\text { Endemicity } \\
\text { (mf } \\
\text { prevalence) }\end{array}$} & \multicolumn{3}{|l|}{ Males } & \multicolumn{3}{|l|}{ Females } \\
\hline & $<25 \%$ & $25-35 \%$ & $\leqslant 35 \%$ & $<25 \%$ & $25-35 \%$ & $\leqslant 35 \%$ \\
\hline $15-19$ years & $27 / 210(228 \cdot 33)$ & $29 / 166(538 \cdot 31)$ & $21 / 102(518 \cdot 81)$ & $22 / 137(328 \cdot 18)$ & $28 / 160(467 \cdot 29)$ & $28 / 115(492 \cdot 96)$ \\
\hline $20-29$ years & $28 / 118(309 \cdot 32)$ & $38 / 128(634.95)$ & $31 / 109(681 \cdot 74)$ & $15 / 92(450 \cdot 80)$ & $36 / 174(518.67)$ & $42 / 133(628 \cdot 50)$ \\
\hline $30-39$ years & $22 / 62(730 \cdot 64)$ & $41 / 98(413 \cdot 20)$ & $36 / 88(957 \cdot 11)$ & $12 / 80(440 \cdot 67)$ & $29 / 134(394.79)$ & $34 / 97(431 \cdot 85)$ \\
\hline $40-49$ years & $16 / 47(585 \cdot 88)$ & $39 / 72(376 \cdot 18)$ & $30 / 56(913 \cdot 93)$ & $13 / 81(363 \cdot 92)$ & $44 / 183(480 \cdot 43)$ & $45 / 142(366 \cdot 42)$ \\
\hline $50-59$ years & $17 / 61(197 \cdot 71)$ & $48 / 87(469 \cdot 33)$ & $58 / 116(559 \cdot 07)$ & $15 / 70(367 \cdot 53)$ & $46 / 179(454 \cdot 70)$ & $64 / 149(583 \cdot 44)$ \\
\hline$\geqslant 60$ years & $14 / 40(104 \cdot 29)$ & $74 / 144(563 \cdot 03)$ & $61 / 103(441 \cdot 11)$ & $17 / 78(485 \cdot 29)$ & $80 / 201(345 \cdot 66)$ & $76 / 171(551 \cdot 21)$ \\
\hline Total & $124 / 538(363 \cdot 67)$ & $269 / 695(503 \cdot 88)$ & $237 / 574(646 \cdot 57)$ & $94 / 538(401 \cdot 74)$ & $263 / 1031(429 \cdot 33)$ & $289 / 807(521 \cdot 18)$ \\
\hline
\end{tabular}

\section{RESULTS}

\section{Patterns of microfilarial aggregation amongst the different strata of the host population}

Table 1 shows the number of microfilaraemic individuals out of the total number of subjects examined and the arithmetic mean of the positive mf loads in the 36 different strata of the population. The 36 values of $k$ estimated by MLM ranged between $0 \cdot 07$ and 0.66 (Fig. 1). The average, common $k, k_{c}$, calculated as the mean of the age-, sex- and endemicityspecific $k$ values, weighted by the reciprocal of each value's estimated variance, was $0 \cdot 30$. According to $\chi^{2}$ tests, the tNBD provided satisfactory fits to the observed data in all but 1 stratum (males aged 15-19 years in high endemicity villages, $k=0 \cdot 29)$. The use of $k_{c}$ to represent $k_{i}$ for each village is supported by the linear regression model, in which parameter $k$ was independent of sex and age of the host, or level of endemicity in the village, either as main effect or included in 2-way interactions (Table 2). It should be noted that the test is approximate, and that a nonsignificant regression coefficient for any of the factors considered only indicates such a factor is likely to be unrelated to $k$.

\section{Predicting the prevalence of high microfilarial loads from the prevalence of microfilaraemia}

Relationships between mean intensity. $M_{i}$ and microfilarial prevalence $\pi_{i}$ at community level. As a first approximation, a linear relationship between the prevalence and mean intensity of $L$. loa microfilaraemia for every village did not provide a very satisfactory fit (coefficient of determination, $R^{2}=0 \cdot 27$ ). To obtain a more robust relationship to be used in the subsequent modelling, we aggregated the villages according to their $\pi_{i}$ values, in the 6 following groups [12-22], [22-27], [27-32], [32-35], [35-38] and $\geqslant 38 \%$, with 6 villages in each group. The linear
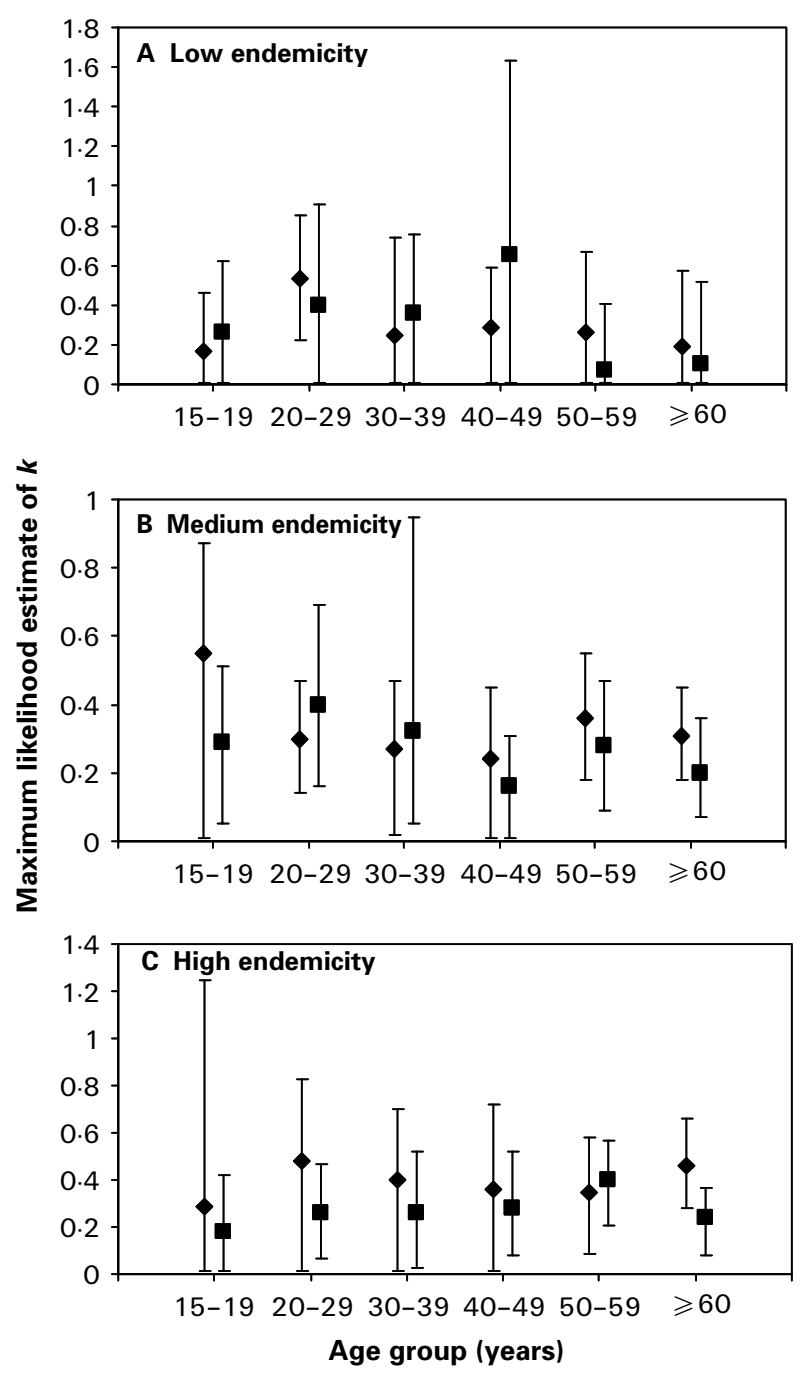

Fig. 1. The degree of microfilarial overdispersion in Loa $l o a$, assessed by the $k$ parameter of zero-truncated negative binomial distribution, according to host age and sex ( : males; $\boldsymbol{\square}$ : females) for different endemicity levels: (A) prevalence of microfilaraemia in the population aged $\geqslant 15$ years, $\pi_{i}<25 \%$, (B) $25 \leqslant\left(\pi_{i}\right)<35 \%$ and $(\mathrm{C})\left(\pi_{i}\right) \geqslant 35 \%$. 
Table 2. Estimates of the linear regression coefficients of the NBD overdispersion parameter $k$ on individual-level variables (host age and sex) and village-level variable (endemicity level) for the distribution of Loa loa microfilarial loads in the Lékié Division, Central Province, Cameroon

(Model is: $k=\beta_{0}+\beta_{1}(\mathrm{Sex})+\beta_{2}$ (Age group) $+\beta_{3}$ (Endemicity $)+\beta_{4}$ (Sex $) \times($ Age group $)+\beta_{5}($ Sex $) \times($ Endemicity $)+$ $\beta_{6}$ (Age group) $\times($ Endemicity $)$. $)$

\begin{tabular}{lrrr}
\hline \hline Variable & Coefficient & 95\% CI & P-value \\
\hline Sex & -0.111 & $-0.411-0.190$ & 0.457 \\
Age group & -0.064 & $-0.131-0.003$ & 0.059 \\
Endemicity & -0.125 & $-0.273-0.022$ & 0.092 \\
Sex $\times$ Age group & 0.004 & $-0.043-0.051$ & 0.860 \\
Sex $\times$ Endemicity & 0.073 & $-0.026-0.171$ & 0.143 \\
Age group $\times$ & 0.024 & $-0.005-0.053$ & 0.102 \\
$\quad$ Endemicity & 0.609 & $0.273-0.944$ & 0.001 \\
Intercept & & & \\
\hline \hline
\end{tabular}

relationship obtained using this grouping $\left(M_{i}=316 \cdot 4 \pi_{i}\right)$ was very similar to that obtained when considering separate communities $\left(M_{i}=314 \pi_{i}\right)$ but considerably improved the fit $\left(R^{2}=0.78\right)$; while the improvement was largely due to a reduction in the number of data points and therefore in the variability of the data, the similarity of the fits suggests that the regression estimate is essentially independent of data grouping (Fig. 2).

Relationship between aggregation parameter $k_{i}$ and mean intensity $M_{i}$. Values of $k_{i}$ assessed for each separate village ranged between 0.09 and 0.81 (Fig. 3). There was no trend in the variation of $k$ with the mean $\mathrm{mf}$ load; the slope coefficient of the log-linear regression $\left(k_{i}=\alpha_{i}+\beta_{i} \log \left(M_{i}\right)\right.$, with $\alpha=-0.31$ and $\beta=0 \cdot 07)$ was not significantly different from zero $(t=0 \cdot 16 ; P<0 \cdot 317)$, supporting the hypothesis that the degree of $\mathrm{mf}$ aggregation is not affected by the level of microfilarial intensity in the community.

Predicting the proportion of heavy microfilarial loads. The predicted prevalence of heavy infections (eqn 1), for $T=8000$ and $T=30000 \mathrm{mf} / \mathrm{ml}$, respectively, were in good agreement with the observed distribution of heavy mf loads (Fig. 4A and B). The model assuming a constant value for the degree of $\mathrm{mf}$ aggregation $\left(k_{c}=0 \cdot 3\right)$ gave satisfactory fits, with non-significant departures between data and models for both the $8000\left(\chi^{2}=39 \cdot 36\right.$, D.F. $=36$, $P<0.66)$ and $30000\left(\chi^{2}=46 \cdot 98\right.$, D.F. $\left.=36, P<0 \cdot 10\right)$ $\mathrm{mf} / \mathrm{ml}$ thresholds. The model assuming a log-linear function, $k_{i}=0.07 \log \left(M_{i}\right)-0.31$ provided similar goodness-of-fit values $\left(\chi^{2}=39 \cdot 01, P<0 \cdot 34\right.$, D.F. $=36$ for $T=8000$; and $\chi^{2}=48 \cdot 11, P<0 \cdot 09$, D.F. $=36$ for $T=30000)$.

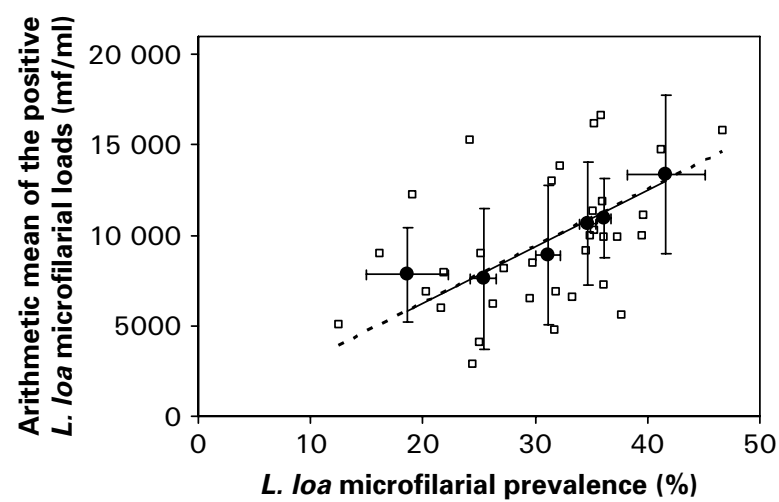

Fig. 2. Relationship between the intensity of microfilarial $(\mathrm{mf})$ loads in the population aged $\geqslant 15$ years in the community and the Loa loa microfilarial prevalence in the same age-group. The squares represent the different villages and the bins (black dots) represent the same villages grouped according to their microfilarial prevalence ( 6 classes, see text). Vertical error bars indicate standard errors of the mean within prevalence bins; horizontal error bars are the standard errors for the $\mathrm{mf}$ prevalence using the normal approximation to the binomial distribution. The dotted line is the regression line with individual village $\left(M_{i}=314 \pi_{i} ; R^{2}=0 \cdot 27\right)$; the solid line is the regression line for the grouped villages $\left(M_{i}=316 \cdot 4 \pi_{i} ; R^{2}=0 \cdot 78\right)$.

\section{ISCUSSION}

\section{Population dynamics insights}

At the time of the surveys, mass ivermectin distribution had not been initiated, and to our knowledge, no significant environmental or ecological changes had taken place in the area. Therefore, our assumption is that the L. loa population was at endemic equilibrium with its human and vector hosts. Our work thus contributes to the characterization of the distribution of L. loa among humans and to highlight its epidemiological implications in natural, nonintervened settings.

One of the main motivations for using the NBD model is that once the degree of overdispersion has been characterized, the theoretical frequency distribution is entirely defined by the arithmetic mean. As the latter is related to the prevalence, it is possible to estimate, from prevalence values, the proportion of hosts harbouring $\mathrm{mf}$ densities above an arbitrary threshold (Guyatt and Bundy, 1991). However, the standard NBD model did not fit well the distribution of the L. loa mf loads probably because in southern Cameroon only $\sim 60 \%$ of the population is genetically predisposed to present with microfilaraemia (Garcia et al. 1999). Instead, the zero-truncated negative binomial distribution, used to describe Wuchereria bancrofti (Pichon et al. 1980; Das et al. 1990; Grenfell et al. 1990) and Brugia malayi (Srividya et al. 1991) $\mathrm{mf}$ densities in some foci of lymphatic filariasis, was found to fit particularly 


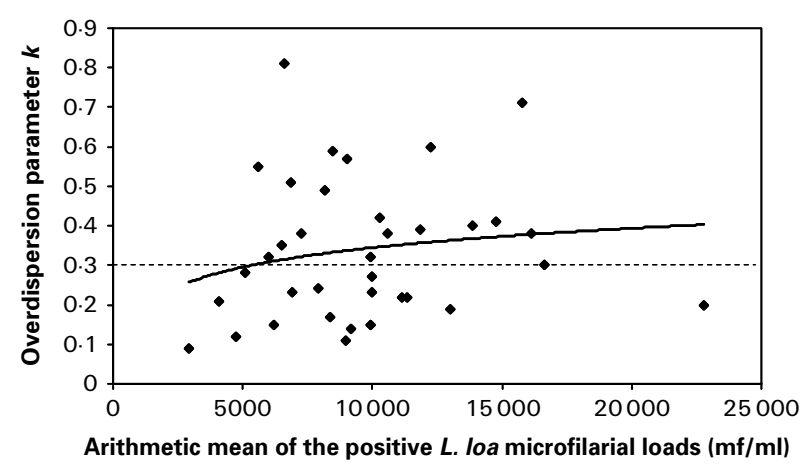

Fig. 3. Relationship between the degree of microfilarial aggregation among the positive individuals aged $\geqslant 15$ years, assessed by the parameter $k$ of zero-truncated negative binomial distributions, and the intensity of microfilarial loads in the community, expressed as the arithmetic mean of the positive microfilarial counts. Solid line: logarithmic function $k_{j}=0 \cdot 07 \log \left(M_{j}\right)-0 \cdot 31$; dashed line: constant function $k_{j}=0 \cdot 3$.

well the distributions of $L$. loa mf loads among the positives.

Some of the models proposed to understand the mechanisms generating overdispersion in hostparasite systems, predict a decrease in the level of aggregation (increase of $k$ ) with host age in the presence of down regulatory density dependence. Such a trend has been taken to indicate operation of parasite-induced mortality of individuals harbouring high parasite densities (Anderson and Gordon, 1982; Pacala et al. 1988) or the development of acquired immunity with age and exposure to infection (Woolhouse et al. 1991; Fulford et al. 1992). We observed a very stable degree of aggregation in the different strata of the population. The fact that we did not observe any trend in the overdispersion pattern with host age does not, however, necessarily imply the absence of processes regulating abundance of L. loa mfs within an individual. Different complex processes, acting simultaneously, may lead to this apparently simple pattern (Duerr et al. 2003). In the case of loiasis, since spontaneous lethal complications are quite uncommon, it seems reasonable to discard a process of parasite-induced mortality of heavily infected hosts in the absence of antifilarial treatment.

\section{Comparison with previous studies on aggregation in filarial infections}

Comparing values of $k$ between different species for which the mean infection intensities are different has some limitations (Taylor et al. 1979; Gregory and Woolhouse, 1993). Nonetheless, the range of overdispersion values observed for $L$. loa was very similar to those observed for other filarial species such as $W$. bancrofti. For this species the value of $k$ has been estimated, using the tNBD model and from independent population samples, as $\sim 0 \cdot 3$ (Pichon et al.
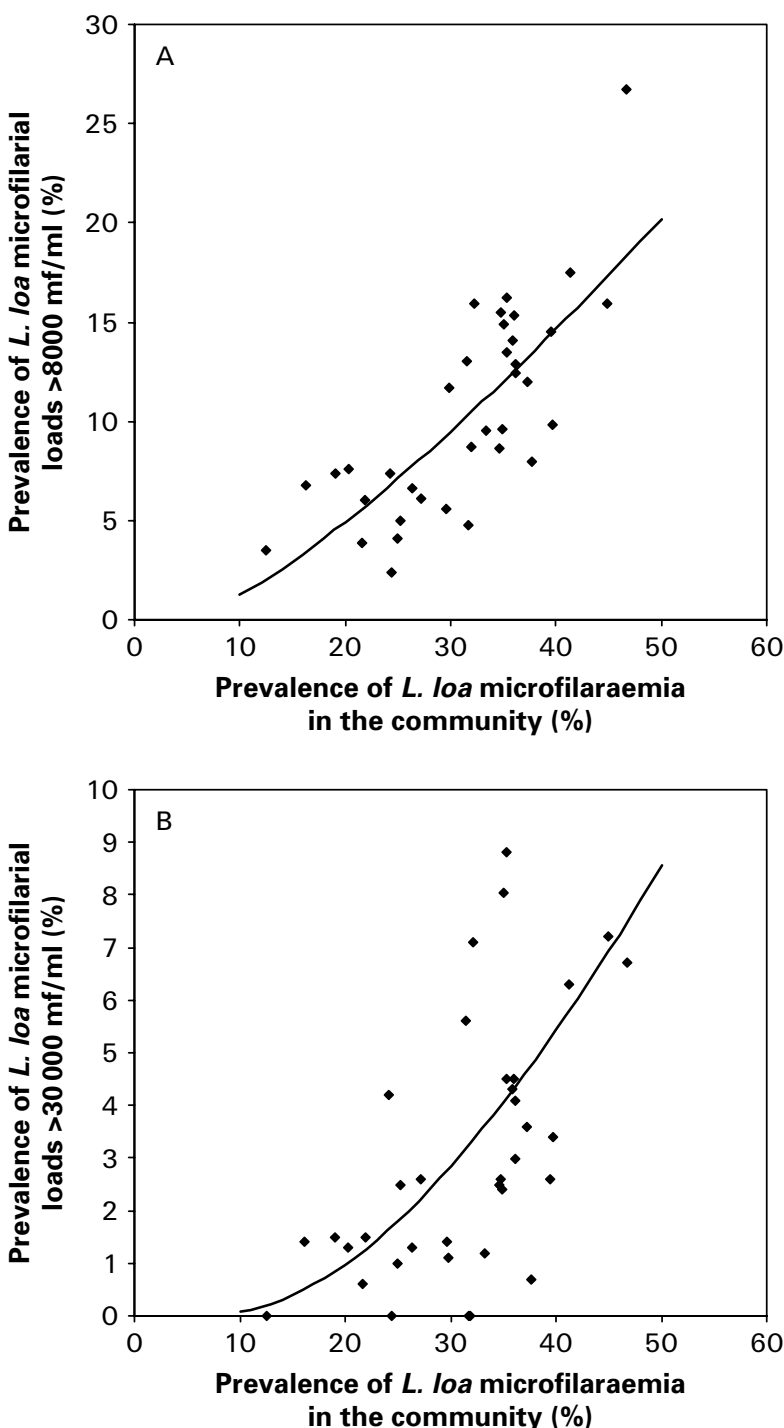

Fig. 4. Relationship between the proportion (in \%) of individuals with microfilarial loads above (A) $8000 \mathrm{mf} / \mathrm{ml}$ and (B) $30000 \mathrm{mf} / \mathrm{ml}$ and the prevalence of Loa loa microfilaraemia among those aged $\geqslant 15$ years in the community. Diamonds: observed values; solid line, predicted relationship with $M_{i}=314 \pi_{i}$ and $k_{c}=0 \cdot 3$.

1980; Grenfell et al. 1990). For O. volvulus, an agestructured model using a zero-inflated NBD yielded $k$ around $0 \cdot 5$ for hosts aged $\geqslant 15$ years (Filipe $e t$ al. 2005).

\section{Predictions of the proportion of the population at risk of $S A E$ s}

We developed a semi-empirical model, aiming at predicting the proportion of the population at risk of post-filaricidal treatment SAEs given the prevalence of microfilaraemia among those aged $\geqslant 15$ years in the community. We developed 2 alternative models, incorporating different assumptions about the relationship $k_{i}\left(M_{i}\right)$ between overdispersion and mean $\mathrm{mf}$ load among the positives in a community: one 
with $k_{i}$ varying with $M_{i}$ and another with a common $k_{i}, k_{c}$. The predictions were not very sensitive to the assumption about $k_{i}$, so adopting a constant value for $k(k=0 \cdot 3)$ would be the most parsimonious and practical approach.

However, if $k_{c} \sim 0.3$ corresponds to endemic equilibrium, the patterns of aggregation are likely to change in communities where large-scale filaricidal treatment is organized. This might constitute a limitation of our present modelling approach, which stands on the analysis of undisturbed populations. Yet, the SAEs are most likely to occur among individuals receiving their first treatment with ivermectin, the post-treatment $\mathrm{mf}$ loads usually remaining below the risk threshold until the next treatment round (Gardon et al. 1997b). A model including a $k_{i}\left(M_{i}\right)$ function would, in principle, be better suited for tracking changes with mean $\mathrm{mf}$ densities following treatments.

The maps provided by the model developed by Thomson et al. (2004) are now used to support APOC's activities, when setting up the CDTI in ivermectin-naïve areas. Such maps give a particularly useful indication of locations where the SAEs surveillance procedures have to be strengthened. As a useful addition to the information generated by the spatial maps, our model would provide information on the proportion of the population at risk of SAEs. The Thomson et al. model gives an indication of location and overall prevalence but not of likely numbers to be affected. If we were able to validate our predictive models at the continental scale across regions where $L$. loa is endemic, either using constant or dynamical overdispersion parameters, we should be able to link the maps of predicted prevalences with maps of population at risk of post-treatment SAEs generated by distributional assumptions. This would constitute a breakthrough regarding the ' $L o a$ challenge' APOC is now facing.

S.D.S.P. is grateful to the Fondation pour la Recherche Médicale and the Fondation Singer-Polignac for financial assistance. J.G. thanks the River Blindness Foundation. The field work was supported by UNDP/World Bank/ WHO Special Programme for Research and Training in Tropical Diseases (Project ID number 950244), and the Institut de Recherche pour le Développement. M.-G.B. and J.A. N.F thank the Medical Research Council (MRC) of the U.K. Dr Paul Clarke helped in the statistical analysis and provided useful comments to the manuscript.

\section{REFERENCES}

Adler, F. R. and Kretzschmar, M. (1992). Aggregation and stability in parasite-host models. Parasitology 104, 199-205.

Akué, J. P., Devaney, E., Wahl, G. and Moukana, H. (2002). Expression of filarial-specific IgG subclasses under different transmission intensities in a region endemic for loiasis. American Fournal of Tropical Medicine and Hygiene 66, 245-250.
Anderson, R. M. (1982). The population dynamics and control of hookworm and roundworm infections. In Population Dynamics of Infectious Diseases (ed. Anderson, R. M.), pp. 67-108. Chapman and Hall, London.

Anderson, R. M. and Gordon, D. M. (1982). Processes influencing the distribution of parasite numbers within host populations with special emphasis on parasite-induced host mortalities. Parasitology 85, 373-398.

Anderson, R. M. and May, R. M. (1985). Helminth infections of humans: mathematical models, population dynamics, and control. Advances in Parasitology 24, 1-101.

Basáñez, M.-G. and Boussinesq, M. (1999). Population biology of human onchocerciasis. Philosophical Transactions of the Royal Society of London, Series B 354, 809-826.

Basáñez, M.-G., Collins, R. C., Porter, C. H., Little, M. P. and Brandling-Bennett, D. (2002). Transmission intensity and the patterns of Onchocerca volvulus infection in human communities. American Fournal of Tropical Medicine and Hygiene 67, 669-679.

Basáñez, M.-G., Remme, J. H., Alley, E. S., Bain, O., Shelley, A. J., Medley, G. F. and Anderson, R. M.

(1995). Density-dependent processes in the transmission of human onchocerciasis: relationship between the numbers of microfilariae ingested and successful larval development in the simuliid vector. Parasitology $\mathbf{1 1 0}$, 409-427.

Basáñez, M.-G., Rodriguez-Perez, M. A., ReyesVillanueva, F., Collins, R. C. and Rodriguez, M. H. (1998). Determination of sample sizes for the estimation of Onchocerca volvulus (Filarioidea: Onchocercidae) infection rates in biting populations of Simulium ochraceum s.l. (Diptera: Simuliidae) and its application to ivermectin control programs. Fournal of Medical Entomology 35, 745-757.

Bliss, C. I. and Fisher, R. A. (1953). Fitting the negative binomial distribution to biological data. Biometrics $\mathbf{9}$, 176-196.

Boulesteix, G. and Carme, B. (1986). Encéphalite au cours du traitement de la filariose à Loa loa par la diéthylcarbamazine. Bulletin de la Société de Pathologie Exotique 79, 649-654.

Boussinesq, M., Gardon, J., Gardon-Wendel, N., Kamgno, J., Ngoumou, P. and Chippaux, J.-P. (1998). Three probable cases of Loa loa encephalopathy following ivermectin treatment for onchocerciasis. American Fournal of Tropical Medicine and Hygiene 58, 461-469.

Boussinesq, M., Gardon, J., Kamgno, J., Pion, S. D. S., Gardon-Wendel, N. and Chippaux, J.-P. (2001). Relationships between the prevalence and intensity of Loa loa infection in the Central province of Cameroon. Annals of Tropical Medicine and Parasitology 95, 495-507.

Carme, B., Boulesteix, J., Boutes, H. and Puruehnce, M. F. (1991). Five cases of encephalitis during treatment of loiasis with diethylcarbamazine. American Fournal of Tropical Medicine and Hygiene 44, 684-690.

Cauchie, C., Rutsaert, J., Thys, O., Bonnyns, M. and Perier, O. (1965). Encéphalite à Loa loa, traitée par 
l'association de cortisone et de carbamazine. Revue Belge de Pathologie et de Médecine Expérimentale 31, 232-244.

Cheke, R. A., Garms, R. and Kerner, M. (1982). The fecundity of Simulium damnosum s.l. in northern Togo and infections with Onchocerca spp. Annals of Tropical Medicine and Parasitology 76, 561-568.

Das, P. K., Manoharan, A., Srividya, A., Grenfell, B. T., Bundy, D. A. and Vanamail, P. (1990). Frequency distribution of Wuchereria bancrofti microfilariae in human populations and its relationships with age and sex. Parasitology 101, 429-434.

Das, P. K., Subramanian, S., Manoharan, A., Ramaiah, K. D., Vanamail, P., Grenfell, B. T., Bundy, D. A. and Michael, E. (1995). Frequency distribution of Wuchereria bancrofti infection in the vector host in relation to human host: evidence for density dependence. Acta Tropica 60, 159-165.

Dobson, A. P. and Hudson, P. J. (1992). Regulation and stability of a free-living host-parasite system Trichostrongylus tenuis in red grouse. 2. Population Models. Fournal of Animal Ecology 61, 487-498.

Duerr, H. P., Dietz, K. and Eichner, M. (2003). On the interpretation of age-intensity profiles and dispersion patterns in parasitological surveys. Parasitology 126, 87-101.

Elliott, J. M. (1977). Some Methods for the Statistical Analysis of Samples of Benthic Invertebrates, 2nd edn. Freshwater biological Association, Scientific Publication 25, Titus Wilson, Cumbria.

Fain, A. (1978). Les problèmes actuels de la loase. Bulletin of the World Health Organization 56, 155-167.

Filipe, J. A. N., Boussinesq, M., Renz, A., Collins, A. C., Vivas-Martinez, S., Grillet, M.-G., Little, M. P. and Basáñez, M.-G. Human infection patterns and heterogeneous exposure in river blindness. Proceedings of the National Academy of Sciences, USA 102, 15265-15270.

Fulford, A. J., Butterworth, A. E., Sturrock, R. F. and Ouma, J. H. (1992). On the use of age-intensity data to detect immunity to parasitic infections, with special reference to Schistosoma mansoni in Kenya. Parasitology 105, 219-227.

Garcia, A., Abel, L., Cot, M., Richard, P., Ranque, S., Feingold, J., Demenais, F., Boussinesq, $M$. and Chippaux, J.-P. (1999). Genetic epidemiology of host predisposition microfilaraemia in human loiasis.

Tropical Medicine and International Health 4, 565-574.

Gardon, J., Gardon-Wendel, N., Demanga-Ngangue, Kamgno, J., Chippaux, J.-P. and Boussinesq, $M$. $(1997 a)$. Serious reactions after mass treatment of onchocerciasis with ivermectin in an area endemic for Loa loa infection. Lancet 350, 18-22.

Gardon, J., Kamgno, J., Folefack, G., GardonWendel, N., Bouchite, B. and Boussinesq, $M$. (1997b). Marked decrease in Loa loa microfilaraemia six and twelve months after a single dose of ivermectin. Transactions of the Royal Society of Tropical Medicine and Hygiene 91, 593-594.

Gregory, R. D. and Woolhouse, M. E. J. (1993). Quantification of parasite aggregation. A simulation study. Acta Tropica 54, 131-139.

Grenfell, B. T., Das, P. K., Rajagopalan, P. K. and Bundy, D. A. P. (1990). Frequency distribution of lymphatic filariasis microfilariae in human populations: population processes and statistical estimation.

Parasitology 101, 417-427.

Guyatt, H. L. and Bundy, D. A. P. (1991). Estimating prevalence of community morbidity due to intestinal helminths: prevalence of infection as an indicator of the prevalence of disease. Transactions of the Royal Society of Tropical Medicine and Hygiene 85, 778-782.

Guyatt, H. L., Bundy, D. A. P., Medley, G. F. and Grenfell, B. T. (1990). The relationship between the frequency distribution of Ascaris lumbricoides and the prevalence and intensity of infection in human communities. Parasitology 101, 139-143.

May, R. M. and Anderson, R. M. (1978). Regulation and stability of host-parasite population interactions. II Destabilizing processes. Fournal of Animal Ecology 47, 219-247.

Pacala, S. W. and Dobson, A. P. (1988). The relation between the number of parasites/host and host age: population dynamics causes and maximum likelihood estimation. Parasitology 96, 197-210.

Pichon, G., Merlin, M., Fagneaux, G., Riviere, F. and Laigret, J. (1980). Etude de la distribution des numérations microfilariennes dans les foyers de filariose lymphatique. Tropenmedizin und Parasitologie 31, 165-180.

Pichon, G., Prod'hon, J. and Rivière, F. (1975). A distribution law for microfilaria ingested by mosquitoes biting human carriers. Preliminary results. Comptes rendus hebdomadaires des séances de l'Académie des sciences 280, 717-719.

Pion, S. D. S., Gardon, J., Kamgno, J., GardonWendel, N., Chippaux, J.-P. and Boussinesq, $M$. (2004). Structure of the microfilarial reservoir of Loa loa in the human host and its implications for monitoring the programmes of Community-Directed Treatment with Ivermectin carried out in Africa. Parasitology 129, 613-629

Poulin, R. (1993). The disparity between observed and uniform distributions - a new look at parasite aggregation. International Fournal for Parasitology 23, 937-944.

Poulin, R. and Morand, S. (2000). Parasite body size and interspecific variation in levels of aggregation among nematodes. Fournal of Parasitology 86, 642-647.

Pugliese, A., Rosa, R. and Damaggio, M. L. (1998). Analysis of a model for macroparasitic infection with variable aggregation and clumped infections. Fournal of Mathematical Biology 36, 419-447.

Quinnell, R. J., Grafen, A. and Woolhouse, M. E. (1995). Changes in parasite aggregation: a discrete infection model. Parasitology 111, 635-644.

Renz, A. (1987). Studies on the dynamics of transmission of onchocerciasis in a Sudan-savanna area of North Cameroon III. Infection rates of the Simulium vectors and Onchocerca volvulus transmission potentials. Annals of Tropical Medicine and Parasitology 81, 239-252.

Sampford, M. R. (1955). The truncated negative binomial distribution. Biometrika 42, 58-69.

Shaw, D. J. and Dobson, A. P. (1995). Patterns of macroparasite abundance and aggregation in wildlife 
populations: a quantitative review. Parasitology 111, S111-S127.

Shaw, D. J., Grenfell, B. T. and Dobson, A. P. (1998) Patterns of macroparasite aggregation in wildlife host populations. Parasitology 117, 597-610.

Srividya, A., Krishnamoorthy, K., Sabesan, S., Panicker, K. N., Grenfell, B. T. and Bundy D. A. (1991). Frequency distribution of Brugia malayi microfilariae in human populations. Parasitology 102, 207-212.

Taylor, L. R., Woiwod, I. P. and Perry, J. N. (1979). The negative binomial as a dynamic ecological model and the density dependence of k. Fournal of Animal Ecology 48, 289-304.

Thomson, M. C., Obsomer, V., Kamgno, J., Gardon, J., Wanji, S., Takougang, I., Enyong, P., Remme, J. H., Molyneux, D. H. and Boussinesq, M. (2004). Mapping the distribution of Loa loa in Cameroon in support of the African Programme for Onchocerciasis Control. Filaria Fournal 6, 7.

Twum-Danso, N. A. (2003). Loa loa encephalopathy temporally related to ivermectin administration reported from onchocerciasis mass treatment programs from 1989 to 2001 : implications for the future. Filaria Fournal 2 (Suppl. 1), S7.

Walker-Deemin, A., Ferrer, A., Gauthier, F., Kombila, M. and Richard-Lenoble, D. (2004). Identification and specificity of a $38 \mathrm{kDa}$ Loa loa antigenic fraction in sera from high-microfilaraemic Gabonese patients. Parasitology Research 92, 128-132.

Winkler, S., Willheim, M., Baier, K., Aichelburg, A., Kremsner, P. G. and Graninger, W. (1999).

Increased frequency of Th2-type cytokine-producing $\mathrm{T}$ cells in microfilaraemic loiasis. American fournal of Tropical Medicine and Hygiene 60, 680-686.

Woolhouse, M. E., Ndamba, J. and Bradley, D. J. (1994). The interpretation of intensity and aggregation data for infections of Schistosoma haematobium. Transactions of the Royal Society of Tropical Medicine and Hygiene 88, 520-526.

Woolhouse, M. E., Taylor, P., Matanhire, D. and Chandiwana, S. K. (1991). Acquired immunity and epidemiology of Schistosoma haematobium. Nature, London 351, 757-759.

Zuidema, P. J. (1971). Renal changes in loiasis. Folia Medica Neerlandica 14, 168-172. 